EPJ Web of Conferences 43, 05008 (2013)

DOI: $10.1051 /$ epjconf/20134305008

(C) Owned by the authors, published by EDP Sciences, 2013

\title{
The potential of 3D radiation-hydrodynamics models for white dwarf asteroseismology
}

\author{
P.-E. Tremblay a and H.-G. Ludwig \\ Zentrum für Astronomie der Universität Heidelberg, Landessternwarte, Königstuhl 12, 69117 \\ Heidelberg
}

\begin{abstract}
White dwarfs with hydrogen-rich atmospheres (DA) are the most abundant of all degenerate objects. In recent years work has been dedicated to increase the accuracy of their model atmospheres. Most notably, convective motions are now treated with 3D radiation-hydrodynamics instead of the standard mixing-length theory. We present and describe selected 3D model atmospheres close and within the instability strip of the pulsating ZZ Ceti white dwarfs. Our 3D simulations depend only weakly on numerical parameters and compared to $1 \mathrm{D}$ models, they provide more realistic determinations of the depth of the convective zone. The 3D structures can then be adopted as input for asteroseismology.
\end{abstract}

\section{INTRODUCTION}

The multidimensional treatment of convective motions in pure-hydrogen atmosphere DA white dwarfs was pioneered by the works of the Kiel group [1-3]. These studies with 2D radiation-hydrodynamics (RHD) models have reported important information about the physical size of the convective flows, the extend of convective overshoot and the RHD effects on the mean spectra. Three-dimensional RHD simulations (hereafter 3D models) recently became accurate enough to be the reference in quantitative spectroscopy [4, 5]. Therefore, Tremblay et al. [6] computed the first four non-gray 3D model atmospheres of DA white dwarfs $\left(12,800<T_{\text {eff }}(\mathrm{K})<11,300\right.$ and $\left.\log g=8\right)$ using the $\mathrm{CO}^{5} \mathrm{BOLD}$ RHD code [7]. The main objective was to look at the 3D effects on the predicted Balmer profiles, and to determine whether the 3D models could solve the long-standing high-log $g$ problem in which the 1D models suggest that cool DA white dwarfs $\left(T_{\text {eff }}<13,000 \mathrm{~K}\right)$ have surface gravities that are much higher than those of hot DA white dwarfs [8-10]. Preliminary results [6], which are now confirmed from improved models presented in this work, have shown that 3D models predict much lower gravities for cool white dwarfs. The mixing-length theory (MLT) [11] is then responsible for the spurious $\log g$ values at cool temperatures and this crude model is therefore unable to adequately represent the transport of convective energy in the photosphere.

In this work, we describe additional properties of our 3D models in relation to the study of $\mathrm{ZZ}$ Ceti white dwarfs. The predictions from ZZ Ceti asteroseismic models are also very sensitive to the parameterization of the MLT theory [12], although they are mostly dependent on the conditions at bottom of the convective zone, a region different to the one surveyed by spectroscopic observations. As a consequence, we compare in this work the physical conditions at the bottom of the convective zone of ZZ Ceti white dwarfs as derived from 1D and 3D models. We show that our 3D simulations could be used as input to derive asteroseismic properties for these objects that do not depend on the mixing-length parameterization.

\footnotetext{
a e-mail: ptremblay@lsw.uni-heidelberg.de
}

This is an Open Access article distributed under the terms of the Creative Commons Attribution License 2.0, which permits unrestricted use, distribution, and reproduction in any medium, provided the original work is properly cited. 


\section{EPJ Web of Conferences}

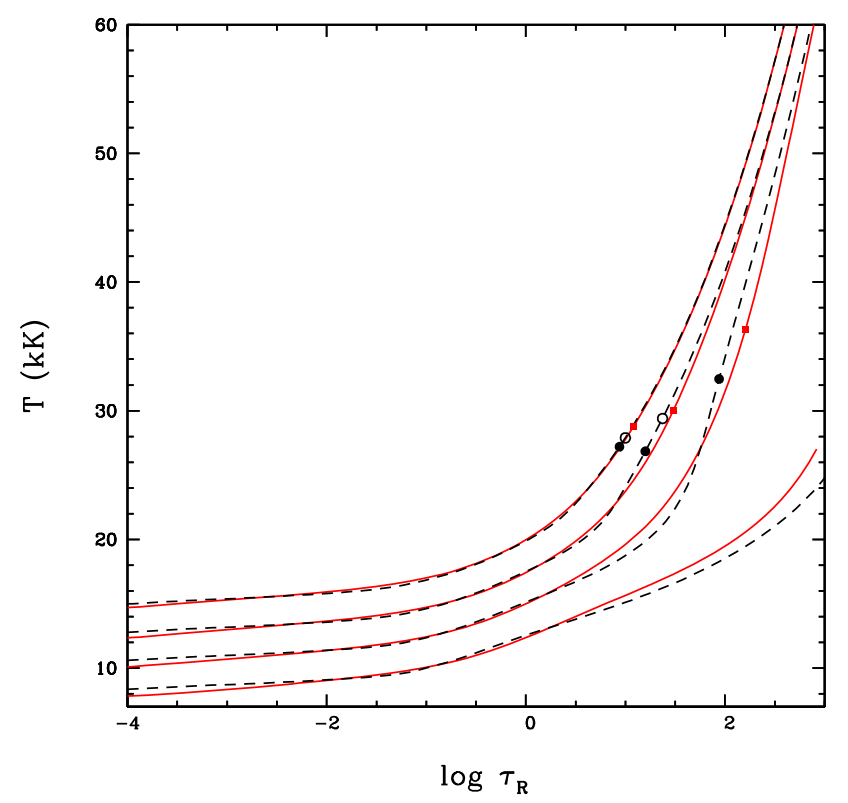

Figure 1. Temperature structures versus $\log \tau_{R}$ for $\langle 3 \mathrm{D}\rangle$ (red, solid lines) and 1D (black, dashed lines) model atmospheres. The temperature scale is exact for the $11,500 \mathrm{~K}$ model, but other structures are shifted by $2 \mathrm{kK}$ for clarity. The filled squares, filled points and open points represent the depth of the convective zone according to the stability criterion $\left(\nabla_{a d}-\nabla>0\right)$ for the 3D, 1D ML2/ $\alpha=0.8$ and 1D ML2/ $\alpha=1.0$ models, respectively. For the $11,500 \mathrm{~K}$ models and the $1 \mathrm{D}$ ML2 $/ \alpha=1.012,000 \mathrm{~K}$ model, the bottom of the convective zone lies at a value larger than $\log \tau_{R}=3$.

\section{SEQUENCE OF 3D MODEL ATMOSPHERES}

For the purpose of this work, we selected four 3D simulations representative of the white dwarfs around the ZZ Ceti instability strip, with $T_{\text {eff }}=11,540,11,946,12,506$ and $13,004 \mathrm{~K}$ at $\log g=8$. We note that the most recent spectroscopic fits of ZZ Ceti white dwarfs [13] place the observed red edge and blue edge of the instability strip at $T_{\text {eff }} \sim 11,100$ and $12,600 \mathrm{~K}$, respectively. Our 3D simulations were computed for small boxes covering the entire atmospheres $\left(-5<\log \tau_{R}<3\right)$. We note that the convective zone is entirely included (in the vertical direction) in the three hotter models, while for the model at $T_{\text {eff }}=11,500$ $\mathrm{K}$, the bottom boundary is open to convection. We relied on the CALYS mini-cluster (Université de Montréal) to perform these calculations. One model takes on average 1 month to compute with 4 cores. Details about the input microphysics and numerical parameters for this improved grid of 3D models will be given elsewhere (Tremblay et al. 2012, in preparation). In brief, the microphysics is the same as in standard 1D models [14] and we have verified that the 3D models were not sensitive to variations of the numerical parameters (e.g., size of the boxes and viscosity), unlike the 1D models which are very sensitive to the parameterization of the mixing-length theory. In Fig. 1 we present $\langle 3 \mathrm{D}\rangle$ structures at $\log g=8$, which are averages in time and space over surfaces of constant Rosseland optical depth, and compare them to $1 \mathrm{D}$ structures, adopting the ML2/ $\alpha=0.8$ parameterization of the mixing-length theory which provides the best match between optical and UV temperatures [10].

Figure 1 also shows the depth of the convective zone, based on the Schwarzschild stability criterion $\nabla_{a d}-\nabla>0$, for the different 3D and 1D models. To show the impact of the mixing-length parameterization, we also add the depth of the convective zone for $1 \mathrm{D}$ ML2/ $\alpha=1.0$ models. For the $T_{\text {eff }}=12,500 \mathrm{~K}$ model, which is very close to the observed blue edge, the depth of the $3 \mathrm{D}$ convective zone is best matched with the ML2/ $\alpha=1.0$ model. This is an interesting result, since the observed blue 


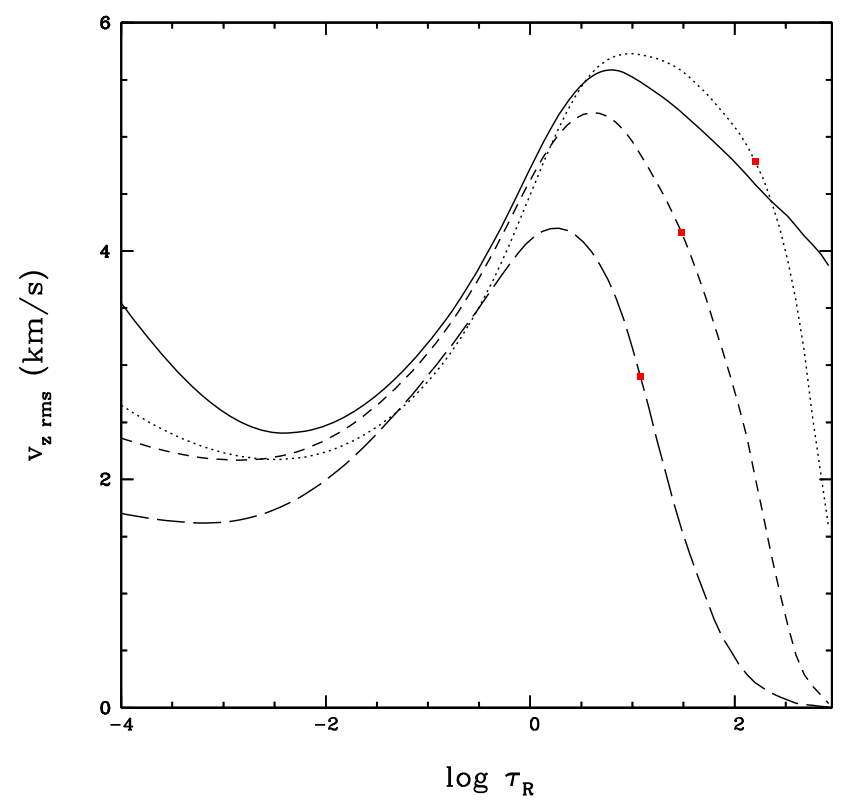

Figure 2. Vertical RMS velocity $v_{z}$ as a function of $\log \tau_{R}$ for the $\langle 3 \mathrm{D}\rangle$ models at $T_{\text {eff }} \sim 11,500$ (solid), 12,000 (dotted), 12,500 (short dashed) and 13,000 K (long dashed). The filled squares represent the depth of the convective zone according to the stability criterion.

edge of the ZZ Ceti instability strip is best reproduced by theoretical models using the ML2/ $\alpha=1.0$ parameterization $[12,17]$. However, we stress that it is necessary to update the observed position of the blue edge with 3D spectra and to update asteroseismic calculations with 3D models to properly compare $1 \mathrm{D}$ and 3D results. We note that for $T_{\text {eff }}=12,000 \mathrm{~K}$, the ML2/ $\alpha=1.0$ model predicts a convective zone that is much deeper $\left(\log \tau_{R}>3\right)$ than what is found with the $3 \mathrm{D}$ simulation. Therefore, the optimal mixing-length value to derive the conditions at the bottom of the convective zone appears to vary significantly within the instability strip.

The stability criterion from which we derived the size of the convective zone might give an incomplete picture. In the case of the 3D simulations, it is not obvious, in comparison to $1 \mathrm{D}$ models, to describe the size of the convective zone. In Fig. 2 and 3, we present the mean vertical velocities, and the ratio of the convective to the total energy flux, respectively. It can be seen from Fig. 2 that an overshoot layer extend, in $\log \tau_{R}$, at least 1 dex and up to 2 dex below the region unstable to convection, a result that was found already from 2D simulations [3]. However, Fig. 3 demonstrates that the overshoot layers transport very little convective energy flux except in the region very close to the bottom of the convective zone. It remains to be seen how this overshoot layer impacts the asteroseismic predictions.

Our simulations with $T_{\text {eff }}<12,000 \mathrm{~K}$ are still convective at the bottom layer, and they may not seem immediately useful for asteroseismic applications. However, [15] have shown that it is possible to match 3D simulations, at their bottom, with 1D structure models, using the ML2/ $\alpha$ value that best match the spatially resolved entropy plateau of the 3D simulations. Another option would be to compute RHD simulations that reach deeper layers.

Finally we note that the observed red edge of the ZZ Ceti instability strip is still not predicted by the theory [12,17]. As a consequence, even in the event that our 3D models would change significantly the position of the bottom of the convective zone compared to current predictions from 1D models, it is unlikely that this would help in any way to explain the red edge. However, since the red edge is thought to be explained from interactions between pulsations and convection [12], it is possible that new quantities drawn from the time-resolved 3D models will help in describing this mechanism. 


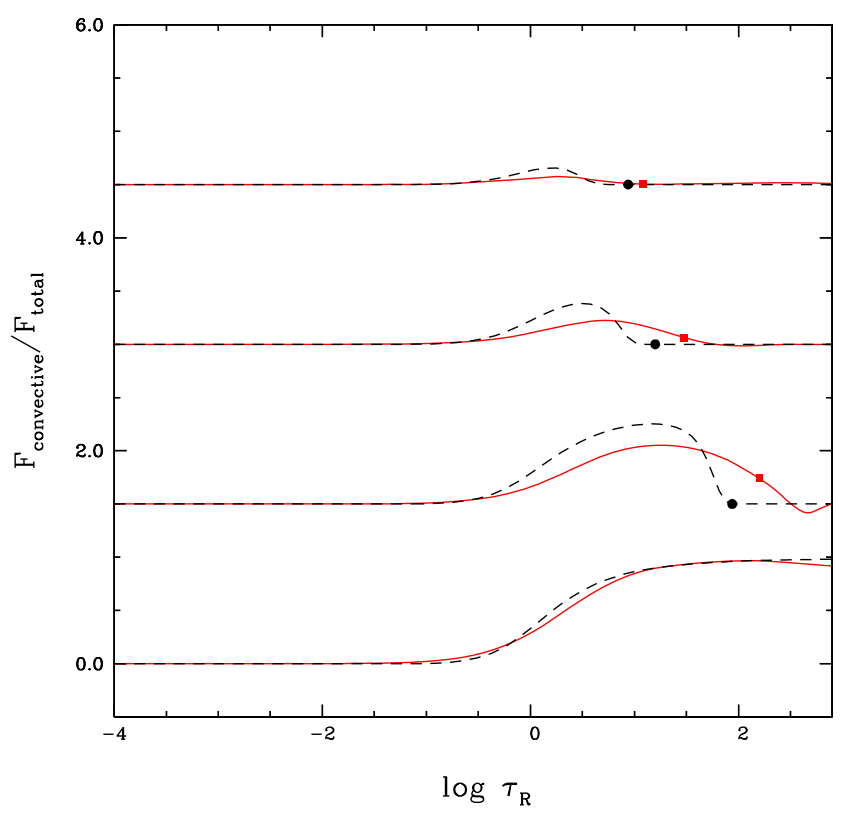

Figure 3. Ratio of the convective to the total energy flux as a function of $\log \tau_{R}$ for our sequence of $\langle 3 \mathrm{D}\rangle$ models (red, solid) and 1D models (black, dotted), with the coolest model at the bottom, and the hottest model at the top. The ratio is exact for the $11,500 \mathrm{~K}$ model, but other structures were shifted by 1.0 for clarity. The filled squares and filled points represent the depth of the convective zone according to the stability criterion for the 3D and 1D models, respectively.

\section{TIMESCALES OF THE 3D SIMULATIONS}

The study of time-resolved white dwarf model atmospheres is of interest for asteroseismology, but it is not easily done with 1D models. It has been understood long ago that convective processes were fairly rapid in ZZ Ceti white dwarfs compared to the typically observed pulsation periods in the range of 100-1000 seconds [12]. As a consequence, non-adiabatic pulsation codes have adopted the concept of instantaneous reaction of the convection to the pulsations [16]. However, the impact of time-resolved convection on the asteroseismology of ZZ Ceti stars has recently been studied using perturbations of the mixing-length equations [17].

In Fig. 4, we present four characteristic timescales as a function of the optical depth in the atmosphere of a 12,000 K white dwarf. The timescales, defined e.g. in [7, 18], have been evaluated under simplifying assumptions and must be taken as order of magnitude estimates only. First of all, the Brunt-Väisälä timescale is related to the period of the oscillations due to the buoyancy force and the spikes indicate the boundaries of the convective zone. The advective timescale is related to the characteristic time for a convective cell to move a distance of one pressure scale height. This timescale does not vary much in the convective zone, with a value below $10^{-1} \mathrm{sec}$.

The Kelvin-Helmholtz or thermal timescale is the thermal energy content (per unit area) divided by the total energy flux and describes the thermal relaxation (through radiative diffusion). The structure of the atmosphere is expected to adjust based on the thermal timescale in the deeper radiative layers where the energy transfer is dominated by the radiative flux. According to Fig. 4, the thermal timescale is of the order of 15 seconds at the bottom of the model. We have verified that in practice, the total flux is stable after a few seconds of simulation in all layers, perhaps due to a correct initial guess of the structure of the deepest layers. 


\section{Ageing Low Mass Stars: From Red Giants to White Dwarfs}

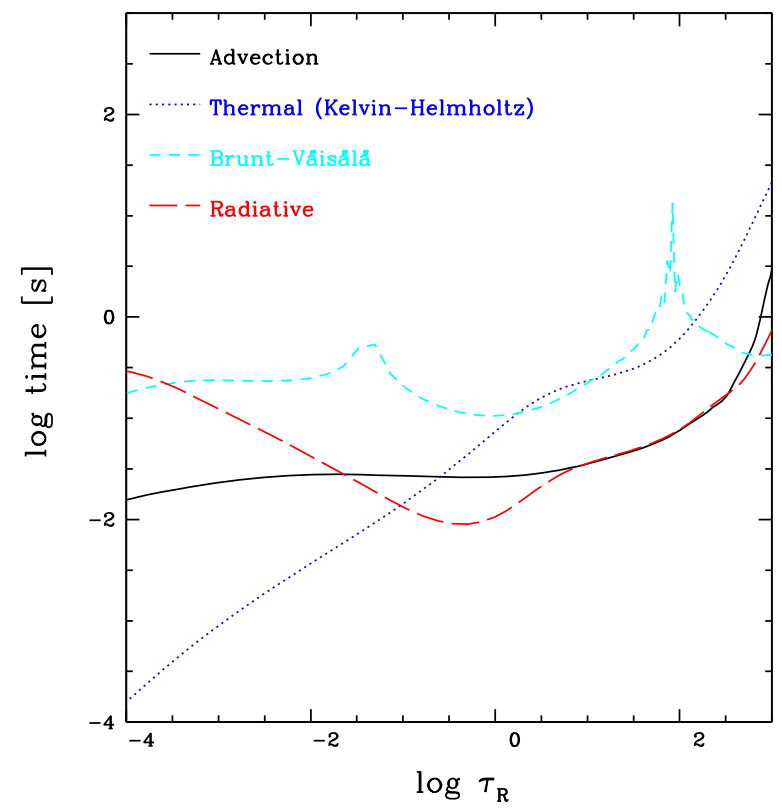

Figure 4. Timescales for the $\langle 3 \mathrm{D}\rangle$ structure of a $12,000 \mathrm{~K}$ DA white dwarf as a function of $\log \tau_{R}$. The different timescales are identified in the legend, and correspond to the advective timescale (solid), the Kevin-Helmholtz timescale (dotted), the Brunt-Väisälä timescale (short dashed) and the radiative timescale (log dashed).

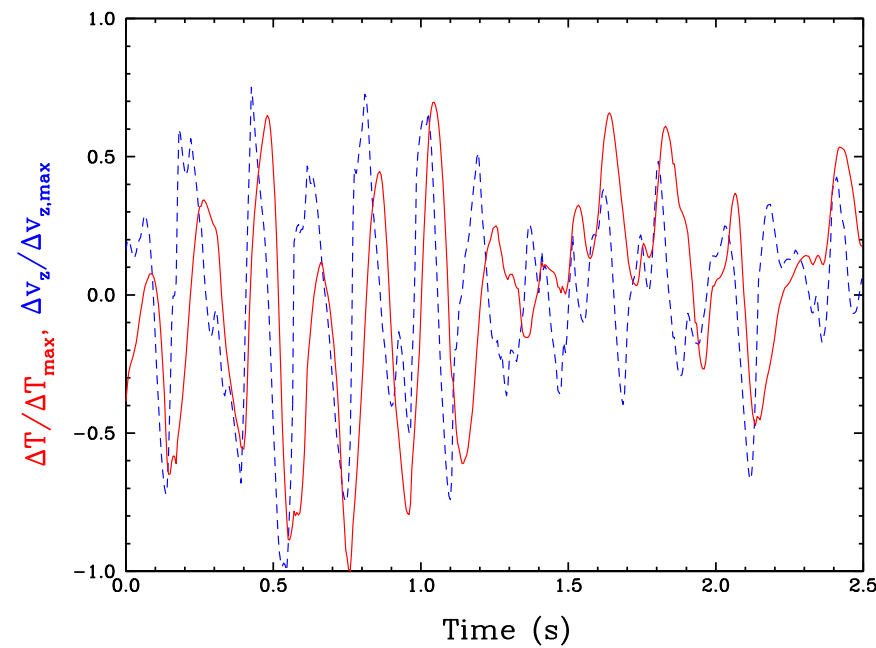

Figure 5. Variation of the mean temperature (red solid line) and the RMS vertical velocity $v_{z}$ (blue dotted line) as a function of time at a geometrical depth corresponding to $\tau \sim 10^{-4}$ in the $12,000 \mathrm{~K}$ simulation. The variations have been normalized to the maximum variation for clarity.

The radiative timescale refers to the characteristic time for the decay of local temperature perturbations through radiative transfer. It is observed that the radiative timescale is of the order of the advective timescale at $12,000 \mathrm{~K}$, and even lower in the photosphere (i.e. the Péclet number is smaller than unity). It implies that radiation is dominant in regulating the formation and evolution of convective cells. We note that in the convective zone, all timescales are lower than one second, which confirms that 
the reaction of the convective zone to changing conditions in the radiative layer just below will be rather instantaneous on the timescales of the pulsations.

The time-resolved quantities (e.g. Reynolds stress or turbulent pressure) that can be extracted from the $\mathrm{CO}^{5} \mathrm{BOLD}$ simulations can be used to understand the interaction of convection with pulsations. We note that most of the our 3D simulations show oscillations, although these are usually short period p-modes with properties that depend of the simulation box boundaries. In Fig. 5, we show as an example the oscillations as a function of time of the RMS vertical velocity and the temperature for one specific layer of the $12,000 \mathrm{~K}$ simulation. These modes, if they exist in real stars, probably can not be observed. However, it shows the potential of utilizing 3D simulations, or perhaps faster $2 \mathrm{D}$ runs, to study the response of convection to forced oscillations.

\section{CONCLUSION}

We presented the properties of four $\mathrm{CO}^{5} \mathrm{BOLD} 3 \mathrm{D}$ radiation-hydrodynamics model atmospheres, characteristic of DA white dwarfs close to or within the ZZ Ceti instability strip $\left(13,000<T_{\text {eff }}(\mathrm{K})\right.$ $<11,500)$. These models are expected to have a precision similar to the standard 1D models and a better accuracy due to the improved treatment of convection. We demonstrated that the properties at the bottom of the convective zone are significantly different between 3D and 1D models, and that one single mixing-length parameterization is inadequate over the full range of the ZZ Ceti instability strip.

It would be adequate at this point to rely on our RHD structures as input for asteroseismic studies. We note that such an application has been performed once [19], although their 2D models predicted a significantly lower convective efficiency than what is found with our 3D models. We believe that the reason for these differences might be that their hydrodynamical code was too much diffusive. Therefore, it is certainly appropriate to update this analysis with our improved 3D models.

\section{References}

[1] Ludwig, H.-G., Jordan, S., \& Steffen, M., A\&A, 284, (1994) 105

[2] Steffen, M., Ludwig, H.-G. \& Freytag, B., A\&A, 300, (1995) 473

[3] Freytag, B., Ludwig, H.-G., \& Steffen, M., A\&A, 313, (1996) 497

[4] Asplund, M., Grevesse, N., Sauval, A.J., \& Scott, P., ARA\&A, 47, (2009) 481

[5] Caffau, E., Ludwig, H.-G., Steffen, M., Freytag, B., \& Bonifacio, P., Sol. Phys., 268, (2011) 255

[6] Tremblay, P.-E., Ludwig, H.-G., Steffen, M., Bergeron, P., \& Freytag, B. A\&A, 531, (2011b) L19

[7] Freytag, B., Steffen, M., Ludwig, H.-G., et al., J. of Comput. Phys., 231, (2012) 919

[8] Bergeron, P., Wesemael, F., Fontaine, G., \& Liebert, J., ApJ, 351, (1990) L21

[9] Koester, D., Kepler, S.O., Kleinman, S.J., \& Nitta, A., J. Phys.: Conf. Ser., 172, (2009) 012006

[10] Tremblay, P.-E., Bergeron, P., Kalirai, J. S. \& Gianninas, A., ApJ, 712, (2010) 1345

[11] Böhm-Vitense, E., ZAp, 46, (1958) 108

[12] Fontaine, G., \& Brassard, P., PASP, 120, (2008) 1043

[13] Gianninas, A., Bergeron, P., \& Ruiz, M. T., ApJ, 743, (2011) 138

[14] Tremblay, P.-E., Bergeron, P. \& Gianninas, A., ApJ, 730, (2011a) 128

[15] Ludwig, H.-G, Freyatg, B. \& Steffen, M., A\&A, 346, (1999) 111

[16] Brassard, P., \& Fontaine, G., in Proc. 10th European Workshop on White Dwarfs, eds. J. Isern, M. Hernanz \& E. Gracia-Berro (Dordrecht: Kluwer), 214, (2007) 451

[17] Van Grootel, V., Dupret, M.-A., Fontaine, G., et al., A\&A, 539, (2012) A87

[18] Ludwig, H.-G., Allard, F., \& Hauschildt, P. H., A\&A, 395, (2002) 99

[19] Gautschy, A., Ludwig, H.-G., \& Freytag, B., A\&A, 311, (1996) 493 